\title{
In vivo response to starch-based scaffolds designed for bone tissue engineering applications
}

\author{
A. J. Salgado, ${ }^{1-3}$ O. P. Coutinho, ${ }^{4}$ R. L. Reis, ${ }^{1,2}$ J. E. Davies ${ }^{3}$ \\ ${ }^{1} 3 B^{\prime}$ 's Research Group, Biomaterials, Biodegradables and Biomimetics, University of Minho, \\ Campus de Gualtar, Braga, Portugal \\ ${ }^{2}$ Department of Polymer Engineering, University of Minho, Campus de Azurém, Guimarães, Portugal \\ ${ }^{3}$ Institute of Biomaterials and Biomedical Engineering, Rosebrugh Building, Taddle Creek Road, \\ Toronto, Ontario, Canada \\ ${ }^{4}$ Department of Biology, University of Miho, Campus de Gualtar, Braga, Portugal
}

Received 3 April 2005; revised 14 March 2006; accepted 24 May 2006

Published online 15 November 2006 in Wiley InterScience (www.interscience.wiley.com). DOI: 10.1002/jbm.a.30946

\begin{abstract}
Our purpose was to evaluate the in vivo endosseous response to three starch-based scaffolds implanted in rats $(n=54)$. We implanted the three scaffold groups; a $50 / 50$ (wt \%) blend of corn starch and ethylene-vinyl alcohol (SEVA-C), the same composition coated with a biomimetic calcium phosphate (Ca-P) layer (SEVA-C/ $\mathrm{CaP}$ ), and a 50/50 (wt \%) blend of corn starch and cellulose acetate (SCA), all produced by extrusion with blowing agents, into distal femurs proximal to the epiphyseal plate, for 1, 3, or 6 weeks. Our results showed that at 1 week considerable reparative bone formed around all scaffold groups, although the bone was separated from the scaffold by an intervening soft tissue interfacial zone that comprised two distinct compartments: the surface of the scaffold was occupied by multinucleate giant cells and the compartment between these cells and the surrounding bone was occupied by a streaming fibrous-like
\end{abstract}

tissue. The extracellular matrix of the latter was continuous with the extracellular bone matrix itself, labeled positively for osteocalcin and appeared mineralized by backscattered electron imaging. All three scaffolds showed a similar tissue response, with the soft tissue interface diminishing with time. No bone contact was observed with SEVA-C at any time point, only transitory bone contact was observed with SEVA-C/CaP at 3 weeks, but SCA exhibited direct bone contact at 6 weeks where $56.23 \pm 6.46 \%$ of the scaffold surface was occupied by bone. We conclude that all materials exhibited a favorable bony response and that the rapidly forming initial "connective tissue" seen around all scaffolds was a very early form of bone formation. (C) 2006 Wiley Periodicals, Inc. J Biomed Mater Res 80A: 983-989, 2007

Key words: tissue engineering; in vivo; bone; scaffold; starch

\section{INTRODUCTION}

Contrary to biomaterials approaches, most tissue engineering strategies are reliant upon the use of a temporary biodegradable scaffold ${ }^{1}$ the ideal structural and morphological properties of which have been thoroughly reviewed elsewhere. ${ }^{2-4}$

We have, since 1995, been developing a series of starch-based polymeric systems for a range of biomedical applications, including bone tissue engineer-

Correspondence to: A.J. Salgado; e-mail: asalgado@ecsaude. uminho.pt

Contract grant sponsor: Portuguese Foundation for Science and Technology; contract grant number: SFRH/BD/ $3139 / 2000$

Contract grant sponsor: Ontario Research and Development Challenge Fund

(C) 2006 Wiley Periodicals, Inc. ing scaffolding. ${ }^{5,6}$ Starch is a polyssacharide comprizing $\alpha$-D-glucose units, which can be organized to form two distinct molecules, amylase and amylopectin. $^{7}$ A possible advantage of using polyssacharidebased biomaterials is that they may act as analogs of polyssacharides present in vivo and adopt their roles. ${ }^{8}$ These starch-based polymeric systems are commonly blended with thermoplastic polymers such as cellulose acetate (CA) or ethylene-vinyl alcohol $(\mathrm{EVOH})$, to better resist thermomechanical degradation, and make them less brittle and more easily processed. ${ }^{7}$ Starch, as a naturally occurring material obviates some of the drawbacks of the synthetic aliphatic polyesters, such as poly(lactic acid), poly(glycolic acid), poly(caprolactone), and their copolymers, which release degradation products, including acidic by-products, that may trigger an inflammatory response and jeopardize integration by the host tissue. ${ }^{9,10}$ In fact previous work conducted 
by our research group has shown that these materials were biocompatible after in vitro and in vivo assays (up to 12 weeks) ${ }^{11}$ and exhibited an improved response when directly compared to PLA. ${ }^{12}$ One of the possible causes of this favorable in vivo response could be the enzymatic degradation products of these materials, which are mainly glucose or glucose derivatives, $^{13}$ although we recognize that longer term degradation studies are necessary to track the biological sequelae of complete degradation.

In vitro experiments have also shown that the degradation products of starch-based scaffolds are not harmful to the cells, and human osteoblasts could populate their surfaces and elaborate extracellular matrix. ${ }^{14-16}$ These starch scaffolds were based on blends of starch with $\mathrm{CA}$ or $\mathrm{EVOH}$, and were obtained by using a methodology based on extrusion with blowing agents. Previous characterization of the latter disclosed porosities between 50 and 70\% and pore sizes in the $200-900 \mu \mathrm{m}$ range, ${ }^{6,15,16}$ exhibiting at the same time a weight loss of about $20 \%$ during the first 10 days of in vitro degradation, degrading at a slower rate thereafter. ${ }^{6}$

Given these encouraging in vitro results, the objective of the present work was to evaluate the in vivo response of three starch-based scaffolds, mentioned in the previous paragraph. The scaffolds were composed of a blend of starch with EVOH (SEVA-C) or with CA, and processed, as previously described, by extrusion with blowing agents. ${ }^{6}$ A third group of SEVA-C scaffolds coated with a biomimetic calcium phosphate (Ca-P) layer was also used as our previous studies had shown that the functional osteogenic phenotype of an osteoblast cell line population was up regulated by these coatings. ${ }^{16}$

Our results show that all the tested groups exhibited biocompatible behaviour, as judged by a lack of a perceived inflammatory response, although bone contacted the SCA material to a greater extent than the other two materials. We also report that the apparent fibrous tissue forming rapidly around the implanted materials positively labeled with a monoclonal antibody for osteocalcin, suggesting that this early reparative tissue was an immature form of bone tissue.

\section{MATERIALS AND METHODS}

\section{Scaffolds production}

Scaffolds used in the present work were composed of: (i) a 50/50 (wt \%) blend of corn starch with EVOH (SEVAC) or (ii) a 50/50 (wt \%) blend of corn starch with CA (SCA), both obtained from Novamont, Italy. A third group of scaffolds based on SEVA-C coated with a CaP layer (SEVA-C/CaP), produced by a biomimetic route, was also used. Scaffolds were obtained through processing by extrusion with blowing agents, as previously described. ${ }^{6}$ Briefly, the polymers were previously mixed, with 1 and $2 \%$ (wt \%) of a solid citric acid-based blowing agent (BIH40, Clariant) for SEVA-C and SCA respectively, in a biaxial rotating drum. This mixture was then extruded in a Carvex twin-screw extruder with a 12-mm die, after which the resulting materials were cut in cylinders of $\sim 2.3 \mathrm{~mm}$ diameter by $3 \mathrm{~mm}$ height. ${ }^{17}$ For the biomimetic premineralization of the SEVA-C scaffolds, samples were first soaked in a commercially available sodium silicate solution (Sigma Aldrich) for $24 \mathrm{~h}$, after which samples were allowed to dry and immersed in a simulated body fluid solution for 14 days ( 20 scaffolds $/ 50 \mathrm{~mL}$ of solution), as described by Oliveira et al. ${ }^{18}$

\section{In vivo studies}

\section{Implantation procedure}

Young male Wistar rats $(n=54)$ with a body weight of 125-150 g were purchased from Charles River (Canada), housed in light- and temperature-controlled rooms, and fed a standard diet. The maintenance and use of animals were in accordance with the Canadian Council of Animal Care Guidelines. Bone defects were drilled bilaterally in each distal femur, proximal to the epiphyseal plate, of every rat. The defects were made using a low speed dental drill $(2.3 \mathrm{~mm}$ in diameter) with copious saline irrigation. Previously cut scaffolds were then press fit into the defects. Empty defects with no filling scaffold/material were used as controls.

\section{Histological processing}

Animals were sacrificed after 1,3 , and 6 weeks $(n=18$ / week), and the femurs ( $n=9$ /group) removed. Femurs ( $n=3$ /group) were fixed in neutral formalin, decalcified in a $1: 1$ mixture of $45 \%$ formic acid and $20 \%$ sodium citrate, dehydrated, and embedded in paraffin. Six-micrometer-thick serial sections perpendicular to the long axis of the implant were cut with a Spencer 820 microtome. Sections were then stained with Masson's Trichrome stain, which employs hematoxylin, anilin blue, and biebrich scarlet to selectively stain muscle, collagen fibers, fibrin, and erythrocytes respectively; bone appears blue. ${ }^{19}$ Bone/scaffold contact was expressed as a percentage of the total scaffold surface measured in 10 micrographs from two femurs, using National Institutes of Health (NIH) image software (http://rsb.info.nih.gov/nih-image/Default.html). Three samples/group were not decalcified and used for Xray, while another 3 /group were used for TRAP (tartrateresistant acid phosphatase) staining.

\section{Immunohistochemistry}

For immunohistochemistry ( $n=2$ /group), samples were fixed in buffered picrate formaldehyde at $4^{\circ} \mathrm{C}$ for 3 days, 
after which samples were decalcified in a $10 \%$ EDTA/4\% $\mathrm{NaOH}$ solution for 20 days. Samples were then embedded in paraffin and sectioned as described before. After deparafinization sections were washed in PBS and successively incubated with $0.2 \%$ hyalurodinase (Sigma), $2 \%$ hydrogen peroxide (Sigma), and $1 \%$ bovine serum albumin (BSA) (Sigma) with PBS washes in between. Sections were then incubated with a monoclonal antiosteocalcin antibody (Takara, Japan) for $16 \mathrm{~h}$ in a moisture chamber at $4^{\circ} \mathrm{C}$, at a working dilution of 1:500. For the negative controls the primary antibody was not used, being replaced instead by PBS. After being washed in PBS, sections were further incubated with the secondary antibody system (Amersham Pharmacia Biotech) for $4 \mathrm{~h}$, in the same conditions as previously described, at a working dilution of 1:200. Visualization of the antibody-enzyme complexes was made possible by incubating the sections with PBS containing $0.5 \mathrm{mg} / \mathrm{mL}$ diaminobenzidine and $0.03 \% \mathrm{H}_{2} \mathrm{O}_{2}$ (Sigma). Sections were finally mounted with glycerin jelly and observed under a light microscope.

\section{Back-scattered electron imaging}

Back-scattered electron imaging was used to observe an early connective tissue that was being elaborated around the implanted scaffolds ( $n=1$ /group). Scaffolds were fixed in neutral formalin, washed for $30 \mathrm{~min}$ in running water, dehydrated, embedded in Osteobed ${ }^{\mathrm{TM}}$ resin, platinum sputtered and observed by scanning electron microscopy $(20 \mathrm{kV})$, in backscattered mode.

\section{RESULTS}

All animals recovered uneventfully from the surgery. Histology revealed that all three materials invoked similar tissue reactions with considerable reparative bone being present at 1 week, but separated from the scaffold surface by an intervening soft tissue interfacial zone [Fig. 1(A,B)]. This soft tissue comprised two distinct compartments with the surface of the scaffold material occupied by darkly stained multinucleate giant cells and the compartment between these cells and the surrounding bone occupied by a streaming fibrous-like tissue which stained faintly blue as illustrated in Figure 1(B). This blue stained extracellular matrix could be easily traced through the soft tissue compartment to the bone margin and was continuous with the extracellular bone matrix itself. Specifically, this matrix was seen to surround osteoblasts at the limit of the histologically identifiable bone matrix [Figure 1(B)]. There was no evidence of inflammatory cells within this soft tissue.

All three sample materials exhibited a multinucleate giant cell (MGC) response. TRAP staining (not shown) was negative in all cases. The MGC layer was seen to be continuous over all available scaffold surfaces in the SEVA-C [Fig. 1(C)] and SEVA-C/CaP samples, while only a discontinuous layer of MGCs was seen in the case of the SCA material. In the case of the former two materials, some MGCs were still observed at 6 weeks while in the case of the SCA scaffolds, the interface was occupied by either bone or marrow at 6 weeks [Fig. 1(D)]. The cellular composition of the interfacial marrow, in contact with the scaffold surface, was indistinguishable from the remaining bulk marrow compartment.

With time, as seen in the 3- and 6-week samples, the amount of reparative bone decreased, as could be expected in this essentially diaphyseal implantation site, but the soft tissue interfacial zone also decreased in width and, in the case of the SCA scaffold was eliminated on some surfaces of the scaffold resulting in direct bone/scaffold contact [Fig. 1(E)]. Such bone contact was also seen, to a less obvious extent, in the case of SEVA-C/CaP scaffolds after 3 weeks of implantation, but after 6 weeks there was no bone contact with this scaffold surface. Thus, bone contact was only measured in the SCA samples, at the 6-week time point, as a percentage of total available linear scaffold surface in a total of 20 micrographs, to be $56.23 \pm 6.46 \%$. Bone contact was not seen in the case of SEVA-C samples at any examination time point.

The soft tissue of the interfacial zone at early healing times was of particular interest, since the extracellular matrix appeared to be continuous with that of the surrounding bone matrix, and was therefore also examined by immuno-labeling for osteocalcin and back-scattered electron imaging. The former revealed that the reparative bone was positive for osteocalcin, but that some areas of the soft tissue which were not histologically identifiable as bone, were also labeled [Fig. 2 (B,D,F]. Similarly, BSEI (Fig. 3) showed that this soft tissue compartment adjacent to bone contained electron dense strands continuous with the bony surfaces of similar backscattering properties to the bone matrix itself.

\section{DISCUSSION}

Our results show that the interfacial tissue zone was occupied by an initial streaming fibrous tissue, which contained osteocalcin positive, and mineralized extracellular matrix. This matrix disappeared in time, particularly in the case of the SCA scaffolds, without any evidence of cellular remodeling, and the interface was occupied by bone. We have reported similar tissue in other rapidly healing bony compartments ${ }^{19}$ and believe that this may represent an early, rapidly forming bone matrix similar to that seen in advancing fronts of intramembraneous bone forma- 

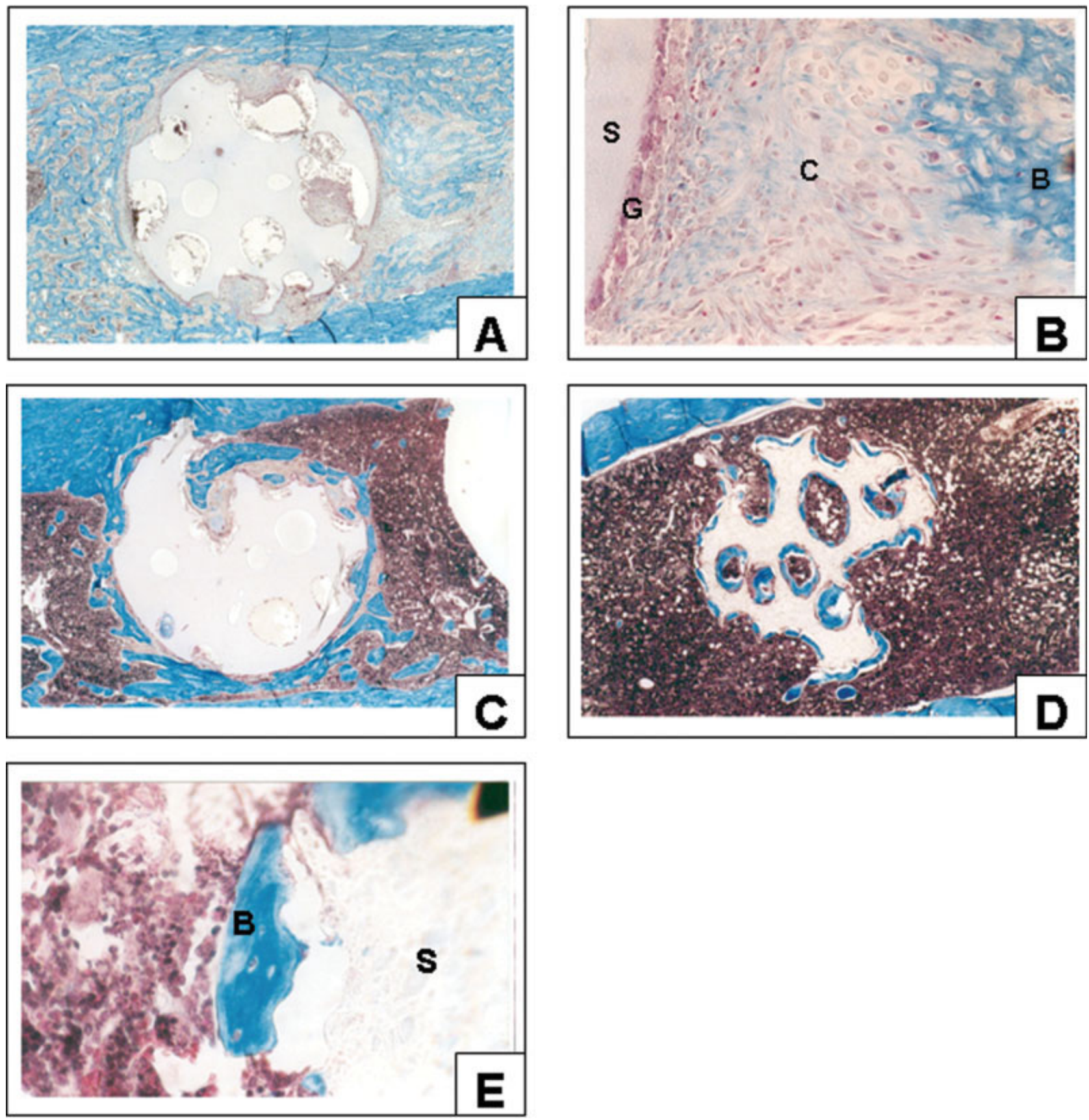

Figure 1. Histological micrographs (Masson's Trichrome staining) of (A,B) SEVA-C/CaP after 1, (C) SEVA-C after 3 and $(\mathrm{D}, \mathrm{E}) \mathrm{SCA}$ after 6 weeks of implantation in rat femora. After 1 week, it was possible to observe the presence of a streaming connective tissue (B) that showed continuity with the surrounding bone trabeculae, and was not present after 6 weeks of implantation (D). Figure D is a representative example of a SCA scaffold after 6 weeks of implantation. As it can be observed the scaffold was very well integrated in a very quiescent surrounding marrow, and thus can be considered as biocompatible. At the same time bone was growing both at the surface and inner pores of the scaffold. When looking at higher magnification (E) it was possible to observe that the bone was directly contacting the scaffolds surface (the existing gap between the scaffold and surrounding bone is an artifact caused by the sectioning of the samples) (B- Bone, CConnective Tissue, G- Multinucleated Giant Cells, S- Scaffold. FW = (a,c,d) 3.5 mm, (b) $351.5 \mu \mathrm{m}$ and (e) $104.6 \mu \mathrm{m}$ ). [Color figure can be viewed in the online issue, which is available at www.interscience.wiley.com.]

tion. The positive labeling with osteocalcin, and back-scattered electron imaging, provides further evidence for this assumption.

Although the healing response to the three scaffold materials employed herein was similar, the dif- ferences in MGC response would seem to correlate with previous studies of protein adsorption to these polymeric surfaces. Thus, our previous work ${ }^{20}$ has shown that SEVA-C-based materials had a more homogeneous protein coating and higher protein 

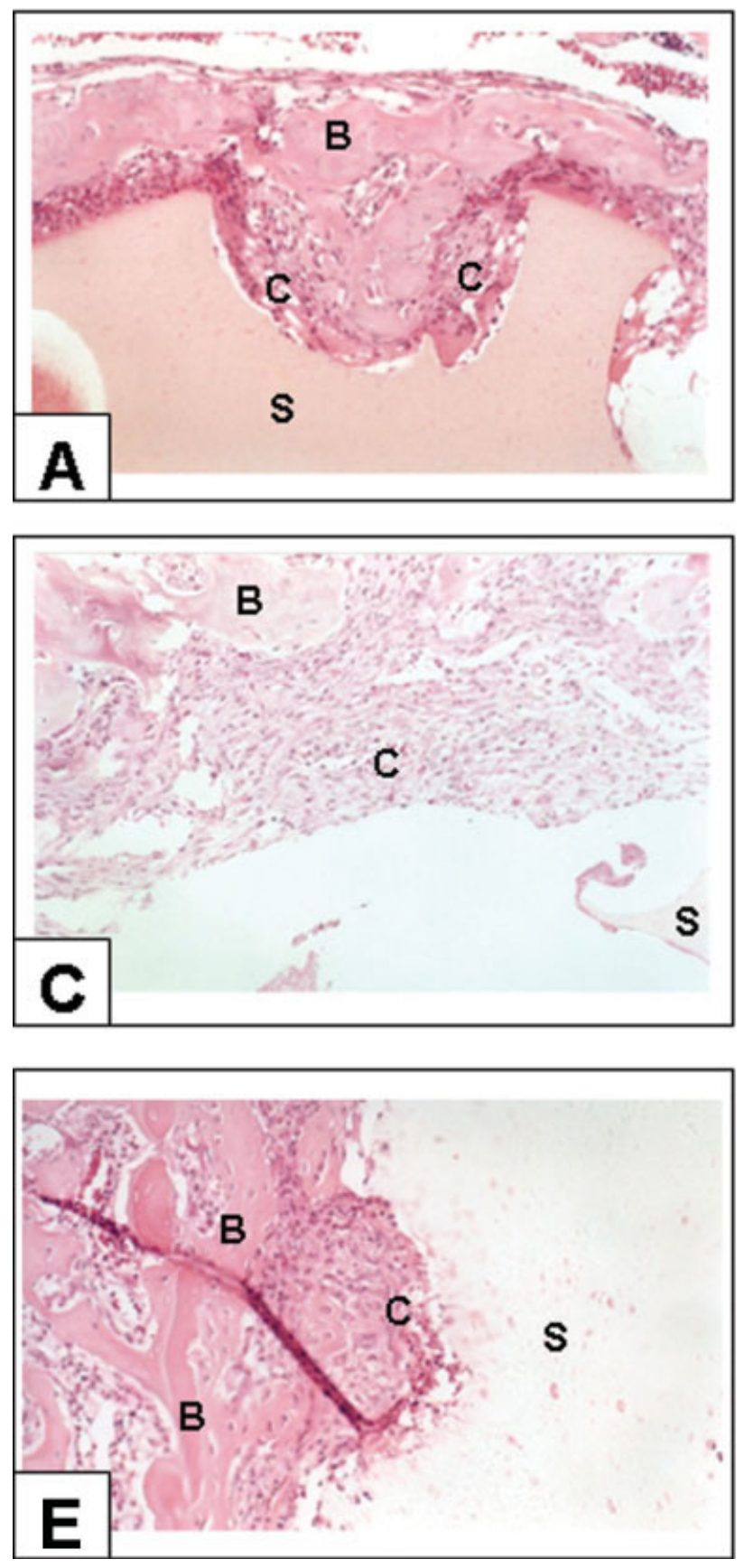
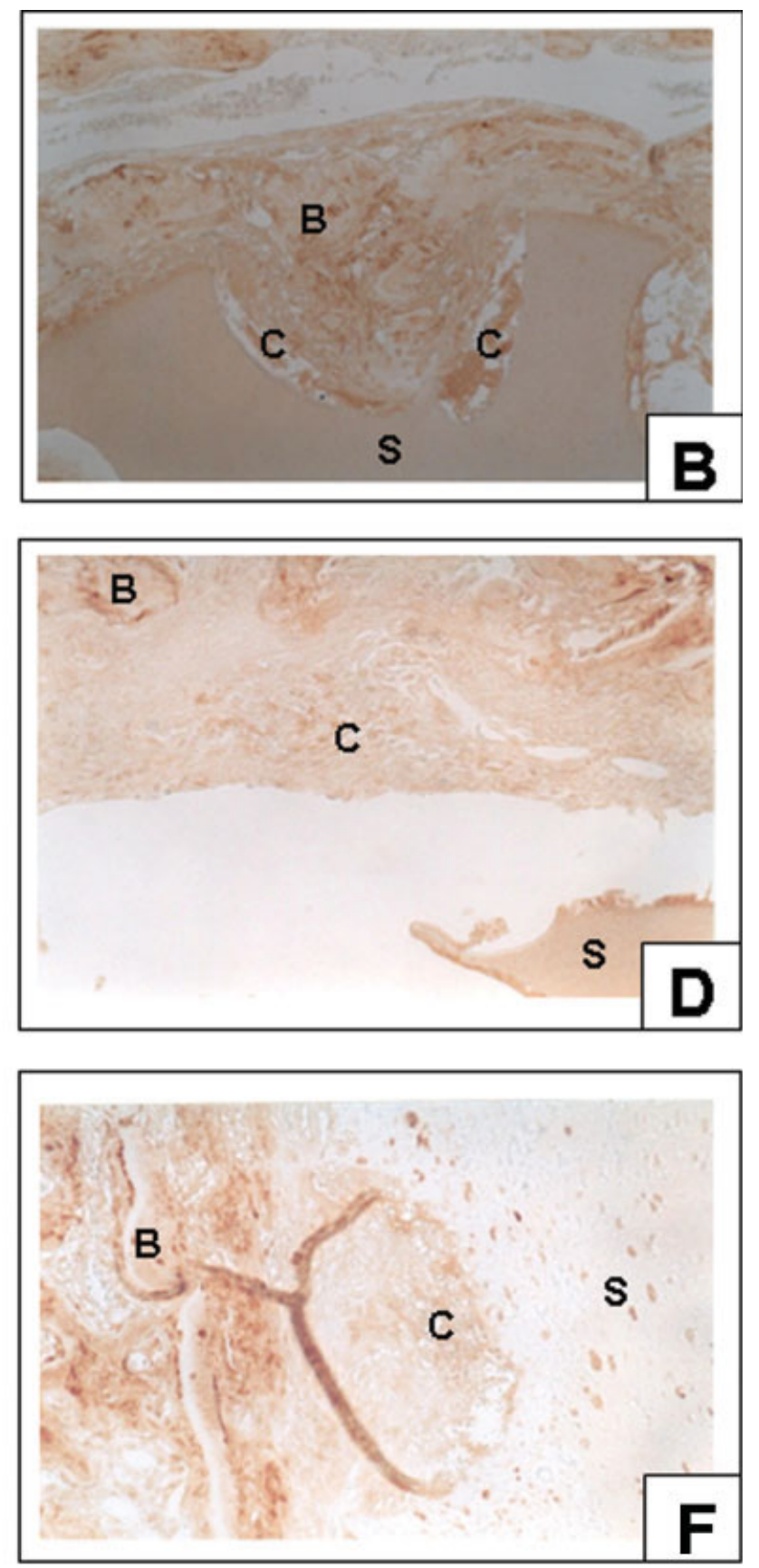

Figure 2. Immunohistochemistry micrographs of SEVA-C (A,B), SEVA-C/Ca-P (C,D), SCA (E,F) scaffolds implanted in rat femora after 1 week. H\&E sections $(A, C, E)$ are shown to illustrate the peri-implant tissue. Samples from all the tested groups labelled positive for osteocalcin $(B, D, F)$, indicating that this early form of connective tissue may be a very early form of bone formation. (B- Bone, C-Connective Tissue, S- Scaffold. FW = A-F) $562.5 \mu \mathrm{m}$ ). [Color figure can be viewed in the online issue, which is available at www.interscience.wiley.com.]

adsorption rates, when compared to SCA surfaces. These differences are probably related with the nature of the two different polymeric blends. While SEVA-C blends are interpenetrating networks, SCA is a nonmiscible blend, resulting in a different distribution of the starch along the surface of the polymeric scaffold. These differences in protein adsorption may explain why the MGC response to the SCA samples was different to the SEVA-C scaffolds.
The tissue reaction to SEVA-C/CaP scaffolds was not what we expected given our previous results obtained in vitro that have shown that this biomimetic coating improved cell adhesion and stimulated osteogenic cell differentiation, as demonstrated by increased ALP activity and bone ECM elaboration. ${ }^{16}$ Nevertheless, the transitory appearance of direct bone contact at the 3-week time point, and its disappearance by 6 weeks indicates not only a favorable 


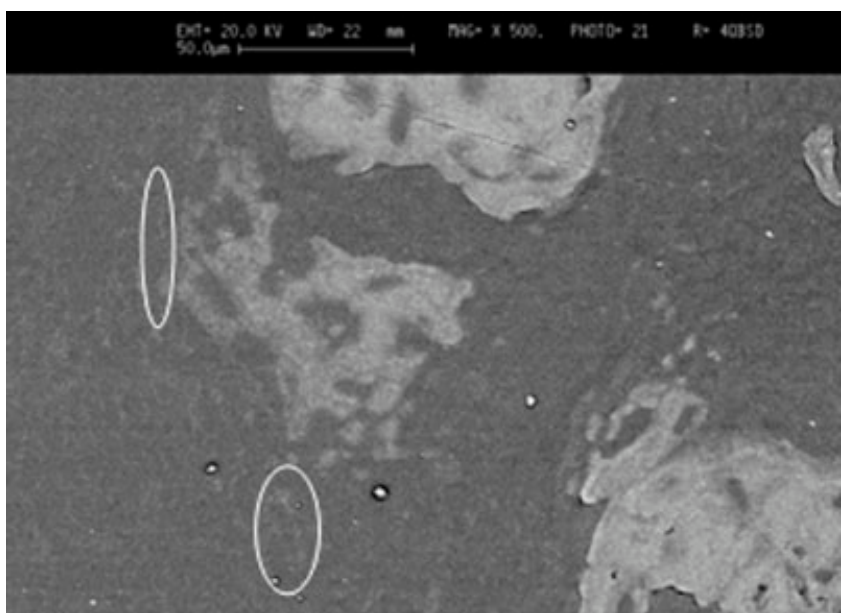

Figure 3. SEM backscattering imaging of the bone/ scaffold interface of SEVA-C/Ca-P scaffold. Strands of connective tissue can be found in the highlighted areas. $(\mathrm{FW}=72 \mu \mathrm{m})$.

bony response to this material, but also the complete remodeling of the reparative bone in this healing compartment and reversion to normal marrow, which can be expected in this diaphyseal site. However, it should also be noted that the in vivo environment is radically different from that found in vitro, and that in vitro results may not be a predictor of in vivo behavior. Furthermore, the limited thickness of the SEVA-C/CaP coating $(10 \mu \mathrm{m})$ and its partially amorphous nature may both result in rapid resorption/dissolution rates, as explained by other authors, ${ }^{21-23}$ and thus explain the similarity in our results with the coated and uncoated SEVA-C scaffolds. Nevertheless, it should be pointed out that although these scaffolds exhibited a MGC response, they were well integrated in the surrounding marrow, indicating a favorable biocompatible response.

Our observations of bone and marrow, contact with the SCA scaffolds are of interest since this thermoplastic biodegradable polymer comprised neither a ceramic filler nor biomimetic coating. Since direct bone/scaffold contact was seen, osteoprogenitor cells must have migrated to the surface of this material, indicating that these scaffolds have osteoconductive properties.

\section{CONCLUSIONS}

We show that the three different starch-based scaffolds (SCA, SEVA-C, and SEVAC/CaP) were well integrated in the defect site and surrounding marrow, indicating their biocompatibility. The major differences found between the 3 tested groups were the degrees of direct bone/scaffold contact, which was only marked in the case of SCA scaffolds. Furthermore, as a result of positive osteocalcin labeling and back-scattered electron imaging, the early connective tissue occupying the bone/scaffold interface can be characterized as an early form of bone.

The authors thank Susan Carter and Feryal Sarraf, from the Faculty of Dentistry at the University of Toronto, for assisting respectively with the surgeries and histological staining.

\section{References}

1. Langer R, Vacanti JP. Tissue engineering. Science 1993;260: 920-926.

2. Agrawal CM, Ray RB. Biodegradable polymeric scaffolds for musculoskeletal tissue engineering. J Biomed Mater Res 2001; 55:141-150.

3. Leong KF, Cheah CM, Chua CK. Solid free form fabrication of three dimensional scaffolds for engineering replacement tissues and organs. Biomaterials 2003;24:3262-2378.

4. Davies JE, Baksh D, Karp JM. Methods in Tissue Engineering, 1st ed. Academic Press: San Diego; 2001. p 333.

5. Gomes ME, Ribeiro AS, Malafaya PB, Reis RL, Cunha AM. A new approach based on injection moulding to produce biodegradable starch based polymeric scaffolds. Biomaterials 2001; 22:883-889.

6. Gomes ME, Godinho JS, Tchalamov D, Reis RL, Cunha AM. Alternative tissue engineering scaffolds based on starch: Processing methodologies, morphology, degradation and mechanical properties. Mater Sci Eng C 2002;20:19-26.

7. Reis RL, Cunha AM.Starch and starch based thermoplastics. In: Jurgen Buschow KH, Cahn RW, Flemings MC, Ilschner B, Kramer EJ, Mahajan S, editors. Encyclopedia of Materials Science and Technology. Amsterdam: Pergamon-Elsevier Science; 2001. pp 8810-8816.

8. Ding Z, Chen J, Gao S, Chang J, Zhang J, Kang ET. Immobilization of chitosan onto poly-lactic acid film surface by plasma graft polymerization to control the morphology of fibroblast and liver cells. Biomaterials 2004;25:1059-1067.

9. Hutmacher DW. Scaffolds in tissue engineering bone and cartilage. Biomaterials 2000;21:2529-2543.

10. Behravesh E, Yasko A, Engel P, Mikos AG. Synthetic biodegradable polymers for orthopaedic applications. Clin Orthop Relat Res 1999;367:S118-S129.

11. Mendes SC, Reis RL, Bovell YP, van Blitterswijk CA, de Bruin JD. Biocompatibility testing of novel starch-based materials with potential application in orthopaedic surgery: A preliminary study. Biomaterials 2001;22:2057-2064.

12. Marques AP, Reis RL, Hunt J. Cytokine secretion from mononuclear cell cultured in vitro with starch-based polymers and poly-L-lactide. J Biomed Mater Res 2004;71:419-429.

13. Azevedo HS, Gama FM, Reis RL. In vitro assessment of the enzymatic degradation of several starch based biomaterials. Biomacromolecules 2003;4:1703-1712.

14. Salgado AJ, Coutinho OP, Reis RL Novel starch based scaffolds for bone tissue engineering: Cytotoxicity, cell culture and protein expression. Tissue Eng 2004;10:465-474.

15. Salgado AJ, Gomes ME, Chou A, Coutinho OP, Reis RL. Hutmacher DW. Preliminary study on the adhesion and proliferation of starch based scaffolds. Mater Sci Eng C 2002;20:27-33.

16. Salgado AJ, Figueiredo JE, Coutinho OP, Reis RL. Biological response to pre-mineralized starch based scaffolds for bone tissue engineering applications. J Mater Sci Mater Med 2005; 16:267-275.

17. Karp JM, Rzeszutek K, Shoichet MS, Davies JE. Fabrication of precise cylindrical three-dimensional tissue engineering scaffolds for in vitro and in vivo bone engineering applications. J Craniofac Surg 2003;14:317-323. 
18. Oliveira AL, Malafaya PB, Reis RL. Sodium silicate gel as a percursor for the in vitro nucleation and growth of a bone like apatite coating in compact and porous polymeric structures. Bioamaterials 2003;24:2575-2584.

19. Rzeszutek K, Sarraf F, Davies JE. Proton pump inhibitors control osteoclastic resorption of calcium phosphate implants and stimulate increased local reapartive bone growth. J Craniofac Surg 2003;14:301-307.

20. Alves CM, Reis RL, Hunt JA. Preliminary study on human protein adsorption and blood cells adhesion to starch based biomaterials. J Mater Sci Mater Med 2003;14: 157-165.
21. Souillac V, Frican JC, Barreile R, Reis RL, Chauveaux D, Baquey C. Starch Based Copolymers as Biomaterials. In Vivo Biocompatibility Study. In Moroni A, Giannini S, editors. Key Engineering Materials. Zurich, Switzerland: Trans Tech Publications; 2001. pp 433-436.

22. ter Brugge PJ, Wolke JG, Jansen JA. Effect of calcium phosphate coating crystallinity and implant surface roughness on differentiation of rat bone marrow cells. J Biomed Mater Res 2002;60:70-78.

23. Ong JL, Villareal DR, Cavin R, Ma K. Osteoblast responses to as-deposited and heat treated sputtered CaP surfaces. J Mater Sci Mater Med 2001;12:491-495. 